\title{
Conocimientos científicos importantes para la conservación y gestión de las tres especies de liebre de la Península Ibérica: deficiencias y retos para el futuro
}

\author{
V. Alzaga ${ }^{1}$, J. Torres ${ }^{1}$, D. Villanúa ${ }^{1}$, A. Cormenzana ${ }^{1}$, I. Leránoz ${ }^{1}$, A. Mateo-Moriones ${ }^{2}$ \\ (1) Gestión Ambiental de Navarra, S.A. C/ Padre Adoain 219, bajo. 31013 Pamplona (Navarra, España) \\ (2) Travesía Iturzar 5. 31395, Iracheta (Navarra, España)
}

* Autor de correspondencia: V. Alzaga [valzagagil@gmail.com]

> Recibido el 31 de enero de 2013, aceptado el 8 de julio de 2013

\begin{abstract}
Alzaga, V., Torres, J., Villanúa, D., Cormenzana, A., Leránoz, I., Mateo-Moriones, A. Conocimientos científicos importantes para la conservación y gestión de las tres especies de liebre de la Península lbérica: deficiencias y retos para el futuro. Ecosistemas 22(2):13-19. Doi.: 10.7818/ECOS.2013.22-2.03

Los principales avances en el conocimiento de las tres especies de liebre presentes en la Península lbérica (Lepus granatensis, Lepus castroviejoi y Lepus europaeus, las dos primeras endémicas de la Península lbérica) tuvieron lugar a finales del siglo XX con la realización de estudios sobre genética, morfología y ecología descriptiva. Durante los 90 y 2000 se profundizó fundamentalmente en aspectos genéticos y evolutivos, en la definición de factores ambientales determinantes de su distribución, en el estado sanitario y en el comportamiento espacial. En lo que respecta a los estudios sobre dinámica poblacional, se dispone únicamente de trabajos locales con metodologías diversas y series de datos generalmente cortas, lo que impide realizar una interpretación conjunta y sólida de los datos. Esta falta de información conlleva que las bases técnicas para determinar una adecuada gestión de las especies sean en la mayoría de los casos insuficientes.

Además, las tres especies se encuentran fuertemente ligadas al medio agrícola y ganadero, por lo que resulta esencial determinar los factores relativos al manejo de estos medios que afectan a las liebres, especialmente debido al declive general de la mayor parte de las especies silvestres ligadas a estos espacios. ¿Será el sector científico-técnico capaz de avanzar en el conocimiento de la dinámica poblacional y el efecto de los distintos tipos de manejo para una mejor gestión de las liebres ibéricas?
\end{abstract}

Palabras clave: Lepus granatensis, Lepus europaeus, Lepus castroviejoi, manejo agrícola, dinámica poblacional

Alzaga, V., Torres, J., Villanúa, D., Cormenzana, A., Leránoz, I., Mateo-Moriones, A. (2013). Scientific knowledge relevant to the conservation and management of the three hare species of the Iberian Peninsula: shortfalls and future challenges. Ecosistemas 22(2):13-19. Doi.: 10.7818/ECOS.2013.22-2.03

The main advances on the knowledge of the three hare species inhabiting the Iberian Peninsula (Lepus granatensis, Lepus castroviejoi and Lepus europaeus, the first two endemic) were carried out in late XXth century, mainly with studies on genetic, morphological and ecological aspects. During the 1990s and 2000s advances were especially related to genetic and evolutionary issues, environmental factors determining species' distributions, sanitary status or spatial behaviour. Available studies about population dynamics are generally local, carried out with different methodologies and short data series, hampering joint data analysis and interpretation. This lack of information leads to insufficient technical background for adequate hare management.

Moreover, the three hare species are linked to agri-environments, so determining the management factors influencing hare populations is essential, especially due to the general decline of most wildlife species linked to these habitats. Will the scientific and technical sectors be able to advance in the knowledge of population dynamics and the effects of different practices in order to improve the management of Iberian hares?

Keywords: Lepus granatensis, Lepus europaeus, Lepus castroviejoi, agri-environmental management, population dynamics

\section{Las liebres en la Península Ibérica}

En la Península lbérica están presentes tres especies de liebre: la liebre europea (Lepus europaeus, Pallas 1778), la liebre ibérica (Lepus granatensis, Rosenhauer 1856) y la liebre de piornal (Lepus castroviejoi, Palacios 1976), siendo estas dos últimas endémicas de nuestro territorio.

Los únicos datos disponibles sobre la evolución de las liebres a nivel estatal son las bolsas de caza, recopiladas por Garrido (2011) para el periodo 2000-2010. Estos datos muestran una tendencia descendente aunque con un aumento considerable de capturas en la última temporada de caza de la que se tiene información (2009-
2010). Carro et al. (2004) analizaron las tendencias poblaciones de las poblaciones de liebre de Navarra y del Parque Nacional de Doñana en los periodos 1991-2003 y 1995-2002, respectivamente, y encontraron una tendencia ligeramente positiva.

Las liebres son especies presa de algunos depredadores de gran importancia para la conservación en la Península Ibérica, como son el águila real Aquila chrysaetos (Fernández y Purroy 1990), el lince ibérico Lynx pardinus (Delibes et al. 2000) o el lobo ibérico Canis lupus signatus (Barrientos et al. 1989). Además gran parte de las poblaciones de liebre peninsulares soportan un aprovechamiento cinegético, siendo esta presión de caza variable a lo largo del gradiente peninsular (Garrido 2011). 
Es importante por tanto conocer la dinámica poblacional de las tres especies y sus principales factores determinantes, para así poder tener bases suficientes para su conservación y gestión, tanto en lo referente al uso y manejo de los espacios que ocupan como a su aprovechamiento cinegético. Por ello nos planteamos como objetivo principal de este trabajo la recopilación de los resultados de los principales trabajos científicos realizados en la Península Ibérica relativos a las tres especies de liebre. Posteriormente se pretende identificar las principales lagunas de conocimiento existentes en la actualidad y extraer los consiguientes retos para el futuro del sector científico-técnico.

\section{Distribución y hábitat}

La distribución de las tres especies de liebre en la Península Ibérica se considera parapátrica (Palacios 1983) (Fig. 1), si bien recientemente Freitas (2006) ha descrito la existencia de hibridación entre liebres ibéricas y europeas en la provincia de Álava.

La liebre ibérica se distribuye desde el extremo más meridional de la Península hasta el occidente de la costa cantábrica, y en la isla de Mallorca, donde se está presente la subespecie $L$. g. solisi (Cabrera 1914, Palacios y Meijide 1979, Duarte 2000, Carro y Soriguer 2002). A nivel general sólo está ausente en la franja que discurre desde el oriente asturiano hasta la costa mediterránea catalana (Duarte 2000). Esta zona está ocupada mayoritariamente por L. europaeus (Ballesteros 2007), que en la Península Ibérica se distribuye al norte del río Ebro, desde el somontano aragonés y los páramos burgaleses y palentinos hasta la frontera con Francia y la costa cantábrica (Gortázar et al. 2007). El área de distribución de la liebre de piornal se encuentra restringida actualmente a unos $5000 \mathrm{~km}^{2}$ de la Cordillera Cantábrica, desde la Sierra de Ancares (entre Lugo y León) hasta la Sierra de Peña Labra (entre Cantabria y Palencia), extensión que supone por tanto toda su área de distribución mundial (Palacios y Meijide 1979, Ballesteros 2003).

La liebre ibérica está fuertemente ligada a los medios agrícolas, al igual que la liebre europea (Duarte y Vargas 1998). Muestra preferencia por los espacios abiertos y zonas de matorral ralo, evitando las superficies boscosas. Aunque en el norte peninsular puede ocupar zonas de pie de monte y pastizales, en la mayor parte de su área de distribución ocupa cultivos de cereal, viñedos, oleaginosas o zonas de pasto para ganado (Duarte 2000, Carro y Soriguer 2002). Un medio especialmente importante para esta especie en el sur peninsular es el olivar, donde puede alcanzar abundancias elevadas (Duarte 2000). Calzada y Martínez (1994) encontraron que la selección de hábitat de las liebres ibéricas variaba a lo largo del año, mostrando preferencia por los terrenos cultivados en invierno y por los barbechos, eriales y alfalfas en verano. Éste, y otros estudios (Alzaga et al. 2011) resaltan la importancia para las liebres ibéricas de las parcelas en descanso escasamente manejadas, de la diversidad en el paisaje agrícola y los mosaicos, y de los elementos naturales en los agrosistemas.
La liebre europea ocupa preferiblemente zonas con cierta ondulación o pendiente (Gortázar et al. 2007), desde campiñas atlánticas con matorral a llanuras cultivadas de carácter mediterráneo de la cuenca del Ebro (Ballesteros 2002).

La liebre de piornal ocupa zonas de montaña atlántica con corta o inexistente sequía estival y con usos del suelo propios de medios pastoreados, caracterizados por un mosaico de pastizales y matorrales (Ballesteros 2003, Acevedo et al. 2007, Ballesteros y Palacios 2009). Estudios realizados mediante parcelas de excrementos (Ballesteros et al. 1996, Ballesteros 2003) señalan su preferencia por los pequeños claros existentes en el interior de las manchas de piornos, así como por las pequeñas zonas quemadas para el ganado de brezal y piornal.

\section{Estudios genéticos}

Bonhomme et al. (1986) y Palacios (1989) mostraron la diferenciación de las tres especies a nivel bioquímico y morfológico respectivamente. Otros autores lo confirmaron posteriormente a nivel de ADN nuclear y mitocondrial (Pérez-Suárez et al. 1994, Alves et al. 2000). Hasta la publicación de estos trabajos la liebre ibérica fue considerada por diversos autores (Flux y Angermann 1990) una subespecie de $L$. capensis.

La Península Ibérica supone una zona de gran interés desde el punto de vista filogeográfico por ser considerada por buena parte del mundo científico como un posible refugio para ciertas especies de liebre durante las últimas glaciaciones (Altuna 1970, Alves et al. 2003 y 2006, Melo-Ferreira et al. 2005, 2007 y 2009, Acevedo et al. 2012).

La liebre europea es una especie relativamente reciente en la mayor parte del continente europeo (López-Martínez 1980, Pierpaoli et al. 1999). La liebre europea que está presente en la Península Ibérica se considera perteneciente a la subespecie $L$. e. europaeus (Palacios 1983 y 1989), si bien importantes diferencias genéticas con otras poblaciones de L. e. europaeus en Bulgaria han sido descritas por Estonba et al. (2006).

Palacios (1976) publicó la descripción de la liebre de piornal, completando y confirmando las apreciaciones de Castroviejo (1970). Inicialmente la especie no fue aceptada por todos los investigadores (Angermann 1983, Schneider y Leipoldt 1983), pero en la actualidad su validez está reconocida gracias a estudios morfológicos, genéticos y bioquímicos (Bonhome et al. 1986, Palacios 1989, Pérez-Suárez et al. 1994).

\section{Reproducción}

En la Península Ibérica sólo existen estudios sobre parámetros reproductivos de la liebre ibérica (ver Tabla 1). En este sentido, Fernández et al. (en Carro y Soriguer 2010) llevaron a cabo un examen detallado del aparato reproductor en las poblaciones de Córdoba y Navarra, con el objeto de determinar la actividad
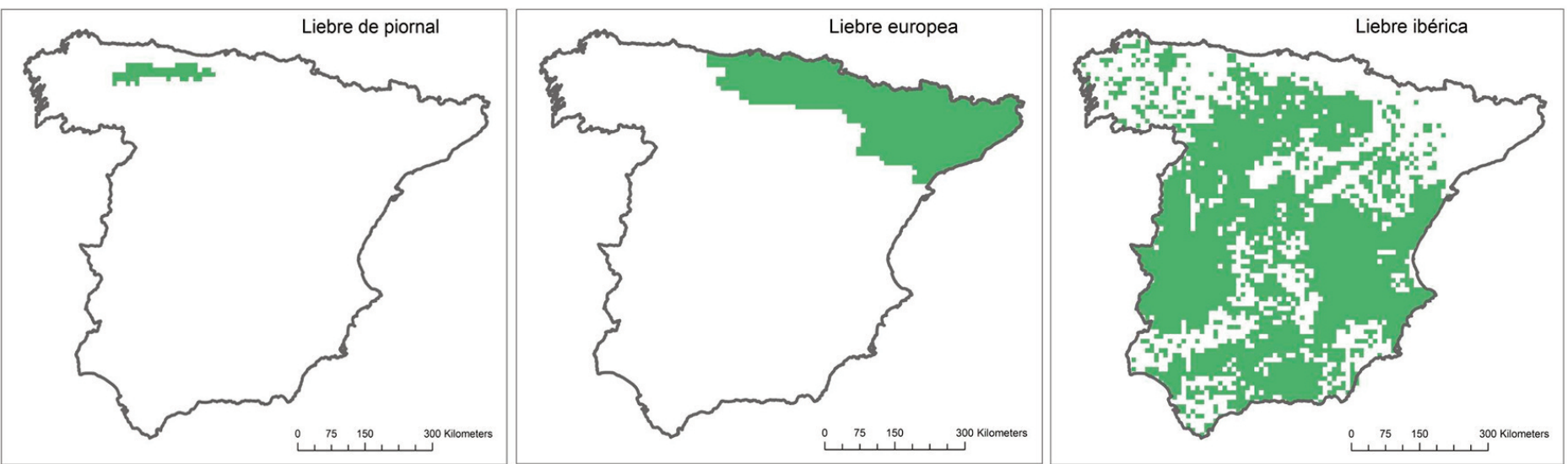

Figura 1. Distribución (en verde) de las tres especies de liebre de la Península Ibérica. Los datos de presencia están referidos a las cuadrículas UTM de $100 \mathrm{~km}^{2}$, adaptados de Palomo et al. (2007). 
reproductiva a lo largo del año y calcular la productividad teórica de las poblaciones estudiadas (ver también Fernández et al. 2008). Anteriormente, Alves et al. (2002) en Portugal y Farfán et al. (2004) en Granada habían realizado un estudio similar (ver Tabla 1)

En el caso de la liebre ibérica, la ausencia de pausa reproductiva definida (Duarte 2000, Alves et al. 2002) complica el uso del conteo de cicatrices uterinas para calcular la productividad de lebratos por hembra y año, tal y como se realiza en la liebre europea (Bray et al. 2003). A pesar de ello, muestras de aparatos reproductivos obtenidas en temporada de caza aportan información útil fundamentalmente cuando se dispone de una serie importante de años y pueden realizarse análisis comparativos (López et al. 1996, Alves et al. 2002, ver Tabla 1).

En cuanto a los factores que influyen en una mayor o menor productividad de las poblaciones, Alves y Rocha (2003) encontraron una menor actividad sexual de los machos en otoño asociada con variables climáticas en el sur de Portugal. Fernández et al. (2010) hallaron máximos en el índice de condición física justo antes del comienzo del pico reproductor, relacionando la acumulación de depósitos de grasa con la gestación.

\section{Estructura poblacional}

Apenas existen trabajos en la Península sobre la estructura de edades de las poblaciones lebreras, dato básico para conocer el tipo de crecimiento poblacional y estimar la tendencia que podrá experimentar una población determinada (Carro y Soriguer 2010). Los valores encontrados en los diferentes estudios realizados en la Península Ibérica se muestran en la Tabla 1. Los porcentajes de juveniles en temporada de caza son en general menores a los obtenidos en otras poblaciones de liebre europea de Europa, por lo que se sugiere que bien la productividad o bien la supervivencia juvenil en las poblaciones ibéricas sea inferior a la de las poblaciones europeas (Pépin 1989, Hansen 1992).

En cuanto a la razón de sexos, diversos trabajos describen un ligero desequilibrio (estadísticamente no significativo) a favor de las hembras en las poblaciones de liebre (Tabla 1). Estos resultados son similares a los obtenidos por otros estudios en el resto de Europa (Jezierski 1965, Frylestam 1979). En el caso de la liebre de piornal los pocos trabajos disponibles (Tabla 1) describen una razón sexual desviada a favor de los machos. Ballesteros y Palacios (2009) sugieren la posibilidad de que los machos puedan ser en este caso más susceptibles a la caza que las hembras.

\section{Dieta}

Las liebres son herbívoros consumidores primarios que, por tanto, concentran la energía de las plantas y facilitan que ésta llegue a niveles tróficos superiores. Lamentablemente no existen trabajos sobre el papel trófico de la liebre en la Península Ibérica. Los estudios sobre dieta de las liebres en la Península Ibérica se limitan al trabajo de Pauperio y Alves (2008) realizado en dos áreas de una zona montañosa del centro de Portugal y al de Carro (2005) en el Parque Nacional de Doñana, ambos relativos a la liebre ibérica. En ambos trabajos las gramíneas se encontraron como base de su dieta, suponiendo siempre más de un $50 \%$ del alimento ingerido, siendo las especies consumidas más frecuentemente Anthoxanthum odoratum, Secale cereale y Agrostis spp. (Pauperio y Alves 2008) o Cynodon dactylon, Hordeum sp., Aeluropus littoralis y Paspalum sp. (Carro 2005). En verano aumentaría la ingesta de otros grupos de plantas, como matorrales e inflorescencias (Pauperio y Alves 2008) y los cultivos de cereal supondrían un importante aporte mientras estos se mantienen verdes (Carro y Soriguer 2002).

No se conocen estudios sobre dieta de la liebre europea en la Península Ibérica. En cuanto a las liebres de piornal, su principal fuente de alimento la constituyen las gramíneas y otras plantas herbáceas fundamentalmente provenientes de los pastizales de montaña (Ballesteros 2003). El rebrote de las formaciones de brezo y piorno tras su quema (actividad ampliamente extendida en los paisajes montañosos donde habita) genera pastos jóvenes que son activamente seleccionados por esta especie (Ballesteros et al. 1996, Acevedo et al. 2007).

\section{Comportamiento espacial y supervivencia}

Carro et al. (2002), y Carro (2005) en el Parque Nacional de Doñana, Bartolomé et al. (2004) en una zona cerealista de Valladolid y, recientemente, Sánchez-García et al. (2012) en León aportaron información sobre supervivencia, área de campeo y factores determinantes del comportamiento espacial de las liebres ibéricas. Los valores de área de campeo se sitúan en torno a 30-40 ha según estos estudios, con variaciones significativas entre individuos. Carro et al. (2002), y Carro (2005) encontraron diferencias, aunque no-significativas, en el área de campeo de machos y hembras (28 ha de media en los machos y 20 ha de media en las hembras). Rodríguez et al. (1997) encontraron valores muy superiores (105-300 ha de media) en liebres traslocadas, por lo que los resultados no

Tabla 1. Principales parámetros poblacionales obtenidos para las tres especies de liebre Lepus spp. en la Península Ibérica.

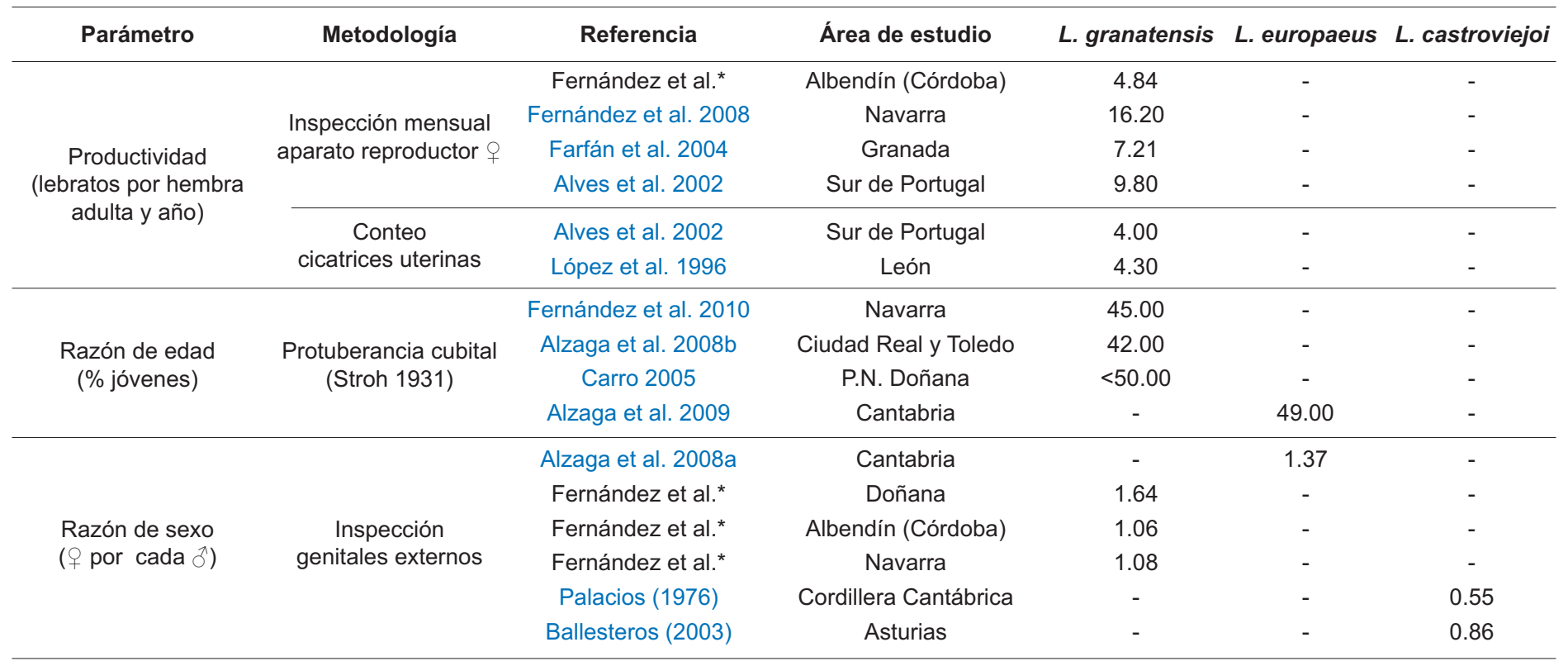

\footnotetext{
* En Carro y Soriguer (2010)
} 
pueden ser extrapolados a poblaciones naturales. Los valores de supervivencia encontrados fueron únicamente de un 20-30 \% en Carro et al. (2002) y de un $13 \%$ en Sánchez-García et al. (2012) en un periodo de estudio inferior a un año y sin tener en cuenta la mortalidad derivada de la caza. Los machos mostraron valores de supervivencia superiores a las hembras, si bien estas diferencias no fueron significativas. Estos resultados se muestran como alarmantemente bajos, siendo justificados por los autores por el bajo número de muestra (Sánchez-García et al. 2012) o por el efecto catastrófico de las inundaciones del periodo de estudio asociado a una alta diversidad de depredadores (Carro et al. 2002). Por tanto, no parece que estos valores sean extrapolables a otras zonas de la Península, tal y como sugieren los propios autores (Carro et al. 2002 y Sánchez-García et al. 2012).

En cuanto a la información disponible para la liebre de piornal, únicamente se dispone de datos de tres liebres radiomarcadas $\mathrm{Ba}$ Ilesteros y Palacios 2009), por lo que no se puede establecer conclusiones sólidas sobre su comportamiento espacial y supervivencia. No se dispone de trabajos similares con poblaciones silvestres de liebre europea en la Península Ibérica.

La creciente importancia de las traslocaciones y repoblaciones como medida de gestión cinegética (Villafuerte et al. 1998) contrasta con la escasa información disponible sobre la supervivencia y viabilidad de estos ejemplares (Alzaga et al. 2008a, Rodríguez Alonso et al. 1997) y sobre sus efectos en las poblaciones de acogida. Los resultados de estos estudios indican valores de supervivencia desde un $2 \%$ a los 20 días post-suelta en el caso de liebres europeas procedentes de granja (Alzaga et al. 2008a) hasta un $36 \%$ y un $52 \%$ (en el periodo de caza y el periodo de veda, respectivamente) en el caso de liebres ibéricas silvestres traslocadas (Rodríguez Alonso et al. 1997). No se dispone de información sobre los resultados numéricos de supervivencia de otras experiencias de repoblación con liebres que se han llevado a cabo en la Península Ibérica ni sobre las posibles experiencias de cría en cautividad que se pudiesen haber llevado a cabo.

En cuanto a índices de abundancia relativa y densidad en las poblaciones de liebre peninsulares, no se encuentran disponibles trabajos con datos actuales, por lo que no les citaremos en la presente revisión. Además el gradiente geográfico y temporal de los mismos es enormemente variable para las tres especies.

\section{Adecuación de hábitat}

En los últimos años se han llevado a cabo varios trabajos sobre modelos de potencialidad de muy diversa índole y objetivo. Acevedo et al. (en Ballesteros y Palacios 2009) diseñaron diversos modelos predictivos a diferentes escalas espaciales. El primero de ellos se realizó a escala de la España peninsular. El factor espacial apareció como determinante en los modelos finales de las tres especies en concordancia con el carácter parapátrico de las especies (Duarte 2000). Las variables climatológicas resultaron también factores relevantes en los modelos de las tres especies. En el caso de la liebre de piornal, las variables topográficas aparecieron como factores relevantes en combinación con los usos del suelo, siendo la combinación de la altitud con el paisaje mosaico derivado de la ganadería extensiva de montaña lo que determina en gran medida su distribución actual (Acevedo et al. en Ballesteros y Palacios 2009). Los usos del suelo también aparecieron como decisivos sobre todo en el caso de la liebre ibérica y la liebre europea, especies ligadas a espacios agrícolas (Duarte y Vargas 1998, Ballesteros 2002).

Los mismos autores desarrollaron otros modelos predictivos a escala más local (Acevedo et al. 2007). Los resultados indicaron que la liebre de piornal es una especie poco plástica y con requerimientos ambientales muy concretos (Ballesteros 2000 y 2003). Además se puso de manifiesto la escasa interconexión de las zonas favorables para la especie, por lo que se sugirió como una buena herramienta de gestión el manejo de los corredores.

Modelos predictivos comparando la liebre de piornal y la liebre europea desarrollados por Acevedo et al. (en Ballesteros y Palacios
2009) en Cantabria indicaron una mayor favorabilidad de la zona de estudio para la liebre europea en comparación con la mostrada para la liebre de piornal. En todo caso, las áreas de potencial convergencia de las dos especies se encontraron ambientalmente más próximas a las zonas adecuadas para la liebre de piornal, por lo que, en caso de establecer relaciones de competencia, la liebre de piornal contaría, a priori, con mayores ventajas adaptativas.

Farfán et al. (2012) modelizaron la favorabilidad ambiental para la liebre ibérica en Andalucía en dos décadas del siglo pasado a partir de datos de bolsas de caza (años 90) y de un mapa de clasificación de terrenos según la abundancia de la especie (años 60). Este estudio muestra cómo las áreas favorables para la especie han evolucionado sustancialmente entre estos dos periodos. Según estos autores la liebre ibérica en Andalucía se habría beneficiado de algunos de estos cambios (ver también Acevedo y Delibes-Mateos 2013), como el incremento de la heterogeneidad de cultivos o de la superficie de cultivos leñosos de secano y herbáceos de regadío en este área (Farfán et al. 2012).

Este tipo de modelos también se ha empleado para elaborar modelos climáticos que permitan, por un lado, explicar la filogeografía de las especies en la Península y, por otro, predecir futuras distribuciones de las especies bajo un escenario de cambio climático (Acevedo et al. 2012). En este trabajo los modelos resultantes predicen una expansión futura de la liebre ibérica hacia el norte y un incremento de la probabilidad de presencia en su rango de distribución actual en un escenario de cambio climático (Acevedo et al. 2012).

\section{Estado sanitario}

En cuanto al estado sanitario de las liebres peninsulares, se han realizado muy pocos trabajos en comparación con el resto de países europeos, siendo éstos generalmente de carácter local. El síndrome de la liebre parda es una enfermedad que ha sido identificada como responsable del declive de varias poblaciones de liebre en Europa (Alzaga 2010). Sin embargo en la Península Ibérica hasta la fecha se ha descrito únicamente un caso de esta enfermedad en un ejemplar de L. europaeus en el Pirineo aragonés (Gortázar y Fernández de Luco 1995). De forma similar, la brucelosis (causada por Brucella suis y muy importante en las poblaciones de liebre del este de Europa, Pikula et al. 2004) sólo se ha detectado en una ocasión en una liebre europea de la Península Ibérica (Lavín et al. 2006).

Una de las enfermedades de mayor interés para la liebre ibérica es la tularemia, fundamentalmente porque este agente infeccioso (Francisella tularensis), además de causar importantes brotes de mortalidad en la especie en algunas zonas de la meseta norte, ha causado varios brotes en el hombre en los últimos años en Castilla y León, relacionándose algunos de éstos con el contacto con liebres (Eiros-Bouza y Rodríguez-Torres 1998).

Por otro lado, Vizcaíno y Fernández (en Carro y Soriguer 2010) realizaron un importante estudio sobre el estado sanitario de las liebres tanto ibéricas como europeas de Navarra, encontrando resultados negativos para la mayor parte de las enfermedades a priori relevantes para la liebre (virus de la liebre parda, tularemia, brucelosis o toxoplasmosis) y escasas prevalencias de listeriosis, pasterelosis, salmonelosis o pseudotuberculosis. Un trabajo reciente llevado a cabo también en Navarra describe la presencia de ejemplares seropositivos al parásito Toxoplasma gondii en liebres ibéricas (Fernández-Aguilar et al. 2013), sugiriendo la existencia de un posible brote en el año 2009 que habría afectado fundamentalmente a individuos jóvenes. Se desconoce si este agente está detrás del descenso de liebre observado en esta zona desde el año 2008. Una seroprevalencia frente a T. gondii elevada (47.3\%) fue también detectada en las liebres del centro-sur peninsular por Alzaga (2010).

Alzaga et al. (2009) analizaron las variables ambientales, poblacionales e individuales que tienen mayor peso en la determinación de la parasitación de un ejemplar de $L$. europaeus del norte 
peninsular. Por otro lado, Alzaga et al. (2008b), tomando como modelo el binomio galgo-liebre ibérica, encontraron una mayor vulnerabilidad a la depredación de las liebres parasitadas por Taenia pisiformis y por parásitos de la familia Coccidia que las liebres no parasitadas. Otro interesante trabajo con comunidades concretas de parásitos ha sido llevado a cabo por Vizcaíno y Fernández (en Carro y Soriguer 2010) en las poblaciones navarras.

\section{Conclusiones}

La batería de conocimientos científico-técnicos disponibles para las especies de liebre de la Península varía según el área del conocimiento y la especie de liebre objeto de estudio (como ejemplo, se han realizado 26 trabajos científicos sobre liebre ibérica, 9 sobre liebre europea y 7 sobre liebre de piornal). Si bien en general puede considerarse escasa (Ballesteros y Palacios 2009, Alzaga 2010). Todo ello a pesar de tratarse de un género de gran relevancia desde el punto de vista de la conservación (Delibes et al. 2000, BaIlesteros y Palacios 2009, Melo Ferreira et al. 2009) y del que se realiza un aprovechamiento cinegético.

La gestión y conservación de las liebres peninsulares se beneficia actualmente de los resultados de los modelos de favorabilidad, ya que permiten identificar áreas clave de conservación para cada especie. Del mismo modo, los diferentes estudios genéticos realizados permiten, por ejemplo, identificar zonas de hibridación entre especies o áreas clave para la conservación debido a su riqueza genética. Además el conocimiento sobre comportamiento espacial aporta información útil para la protección y creación de espacios favorables para las tres especies, identificando los manejos agrícolas, ganaderos o forestales que favorecen a las liebres en cada etapa del año (Ballesteros y Palacios 2009, Alzaga et al. 2011).

La realización de un mínimo seguimiento sanitario de las poblaciones de liebre resulta esencial para evaluar las implicaciones de las enfermedades en la dinámica de las poblaciones y para valorar problemáticas asociadas a ciertos modelos o manejos cinegéticos (Villanúa et al 2008). Además, teniendo en cuenta que algunas de las enfermedades que afectan a las liebres son zoonosis, una monitorización resulta también interesante en el campo de la salud pública.

La información disponible para las tres especies de liebre presentes en la Península Ibérica puede considerarse insuficiente en determinadas áreas, lo que provoca las siguientes carencias:

- Ausencia de series temporales suficientemente largas de datos referentes al estatus poblacional, reproductivo y sanitario.

- Los estudios locales son realizados con metodologías diversas que imposibilitan la comparación de los resultados entre sí.

- Inexistencia de un método de recogida estandarizado y homogéneo de las bolsas de caza en las diferentes Comunidades Autónomas.

- Ausencia de estudios que valoren las implicaciones de los usos y manejos agrarios en las poblaciones lebreras y que evalúen la eficacia de los diferentes modelos y medidas de gestión cinegética que se llevan a cabo en la Península.

Estas lagunas de información impiden que se puedan realizar trabajos sobre dinámica poblacional sólidos de las tres especies de liebre peninsulares y que conozcamos su estado de conservación y tendencia poblacional, al contrario que ocurre en el caso de otras poblaciones de liebre europeas (Schmidt et al. 2004, Kauhala et al. 2005, Reynolds et al. 2006). Para realizar este tipo de trabajos es imprescindible primeramente un sistema de recogida de datos estandarizado y fiable.

\section{Propuestas para el futuro}

A la vista de lo expuesto en esta revisión, los retos que se plantean para el futuro en lo que respecta a las liebres peninsulares son múltiples:

\section{Sobre su estado poblacional:}

1 Establecimiento de sistemas de monitorización de las poblaciones de liebre mediante metodologías estandarizadas adecuadas para la especie (Barnes y Tapper 1985) y consecución de un acuerdo para su empleo por parte de las Comunidades Autónomas con presencia de poblaciones de liebre.

2 Identificación de un método sencillo y fiable de recogida de datos de bolsas de caza por parte de las Comunidades Autónomas y búsqueda de consenso para su empleo a escala peninsular.

3 Seguimiento de la estructura de determinadas poblaciones de liebre que se consideren representativas de las diferentes situaciones poblacionales de la Península Ibérica. El registro de esta información durante las primeras jornadas de caza permite estandarizar la información y tener capacidad de actuación sobre la presión de caza a ejercer durante el resto de la temporada. Un posible método sencillo y fácil para realizar este registro está basado en la presencia de la protuberancia cubital en los ejemplares jóvenes (Stroh 1931).

4 Ampliación del conocimiento, mediante trabajos experimentales con animales en cautividad, que evalúen: 1) el comportamiento reproductivo de las liebres a lo largo del año, con el objetivo de valorar la aplicación de los métodos de estimación del éxito reproductivo empleado en otras regiones europeas; y 2) los efectos que algunas de las enfermedades que afectan a la liebre pueden tener sobre sus estructuras poblacionales (por ejemplo, la toxoplasmosis en Navarra).

5 Dada la falta de trabajos que aborden la influencia de los depredadores sobre la dinámica poblacional de las liebres peninsulares, se hacen necesarios estudios de este tipo, así como el análisis de la eficacia real de las medidas de control de depredadores tan ampliamente demandadas en el sector cinegético (Díaz-Ruíz y Ferreras 2013).

\section{Sobre su estado sanitario y genético:}

1 Establecimiento de un sistema de seguimiento sanitario en poblaciones representativas del gradiente peninsular. Este seguimiento debería contar con un esfuerzo mayor en las zonas donde se han descrito problemas sanitarios de importancia, donde se detecte un importante declive poblacional y donde se realicen traslocaciones o repoblaciones cinegéticas, por el riesgo sanitario que estas prácticas suponen (Villanúa et al. 2008).

2 Realización de un estudio genético en las zonas límite de distribución con el objetivo de determinar la variabilidad genética y la posible presencia de ejemplares híbridos.

\section{Sobre su gestión:}

1 Estudio de la influencia de los diferentes manejos agrícolas, ganaderos y forestales en las poblaciones de liebre con el objetivo de identificar medidas de Buenas Prácticas agrarias y forestales. Una vez establecidas las directrices de mejora de poblaciones para cada uno de los diferentes escenarios existentes, consensuar e integrar estas medidas en los programas de gestión agrícola, forestal y del paisaje de las zonas de mayor vulnerabilidad.

2 Análisis del efecto de la presión cinegética sobre las diferentes poblaciones de liebre con el objetivo de establecer directrices que permitan determinar los ejemplares de una población que pueden ser extraídos sin poner en riesgo su sostenibilidad. Este análisis resulta especialmente necesario en el caso de la liebre de piornal, en la que se cuestiona la posibilidad de su aprovechamiento cinegético debido a su carácter vulnerable ligado fundamentalmente a su limitada área de distribución (Alzaga et al. 2008a).

3 Puesta en valor de las especies cinegéticas por parte de todos los sectores implicados en la conservación, en tanto se trata de especies silvestres base de nuestros ecosistemas y posibles abanderadas de la conservación de los hábitats que ocupan. En este sentido, resulta importante monitorizar y controlar el estatus de conservación de estas especies. 


\section{Referencias}

Acevedo, P., Alzaga, V., Cassinello, J., Gortázar, C. 2007. Habitat suitability modelling reveals a strong niche overlap between two poorly known species, the broom hare and the Pyrenean grey partridge, in the north of Spain. Acta Oecologica 31:174-184.

Acevedo, P., Melo-Ferreira, J. Real, R., Alves, P.C. 2012. Past, present and future distributions of an Iberian endemic, Lepus granatensis: Ecological and Evolutionary Clues from Species Distribution Models. PLOS One 7(12):e51529.

Acevedo, P., Delibes-Mateos, M. 2013. Efectos de los cambios en los usos del suelo en las especies cinegéticas en el sur de España: repercusiones para la gestión. Ecosistemas 22(2):33-39.

Altuna, J. 1970. Hallazgo de una liebre ártica (Lepus timidus) en el yacimiento prehistórico de Urtiga (Guipuzcoa). Munibe 22:165-168.

Alves, P.C., Rocha, A. 2003. Environmental factors have little influence on the reproductive activity of the Iberian hare (Lepus granatensis). Wildlife Research 30:639-647.

Alves, P.C., Branco, M., Matias, O., Ferrand, N. 2000. New genetic variation in European hares, Lepus granatensis and L. europaeus. Biochemical Genetic 38(3-4):87-96.

Alves, P.C., Gonçalves, H., Santos, M., Rocha, A. 2002. Reproductive biology of the Iberian hare, Lepus granatensis, in Portugal. Mammalian Biology 67:358-371.

Alves, P.C., Ferrand, N., Suchentrunk, F., Harris, D.J. 2003. Ancient introgression of Lepus timidus mtDNA into $L$. granatensis and $L$. europaeus in the Iberian Peninsula. Molecular Phylogenetics and Evolution 27:70-80.

Alves, P.C., Harris, J., Melo-Ferreira, J., Branco, M., Suchentrunk, F., Boursot, P., Ferrand, N. 2006. Hares on thin ice: Introgression of mitochondrial DNA in hares and its implications for recent phylogenetic analyses. Molecular Phylogenetics and Evolution 40:640-641.

Alzaga, V. 2010. Ecología de la parasitación de las liebres (Lepus spp.) de la Península Ibérica. Tesis doctoral. Universidad de Castilla-La Mancha, España.

Alzaga, V., Acevedo, P., Gortázar, C. 2008a. Situación actual de la liebre en Cantabria: bases científicas para una adecuada gestión. Locustella 5:28-43.

Alzaga, V., Vicente, J., Villanúa, D., Acevedo, P., Casas, F., Gortazar, C. 2008b. Body condition and parasite intensity correlates with escape capacity in Iberian hares (Lepus granatensis). Behavioral Ecology and Sociobiology 62:769-775.

Alzaga, V., Tizzani, Acevedo, P., Ruiz-Fons, F., Vicente, J., Gortázar, C. 2009. Deviance partitioning of host factors affecting parasitization in the European brown hare (Lepus europaeus). Naturwissenschaften 96(10):1157-1168

Alzaga, V., Villanúa, D., Ros, F., Leránoz, I., Torres, J., Cormenzana, A., González, S., Urmeneta, A. 2011. Influence of farmland management on Iberian hare (Lepus granatensis) space use in a steppe-land habitat. Abstract book of the XXXth IUGB Congress and Perdix XIII, Barcelona 5-9 Septiembre 2011. pp. 141.

Angermann, R. 1983. The taxonomy of Old World Lepus. Acta Zoologica Fennica 174:17-21.

Ballesteros, F. 2000. Técnicas aplicables para la estimación y monitorización de la abundancia de la liebre de piornal (Lepus castroviejoi). Naturalia Cantabricae 1:45-51.

Ballesteros, F. 2002. Lepus europaeus. En: Palomo, L.J. y Gisbert, J. (eds.), Atlas de los Mamíferos Terrestres de España. SECEM, Madrid, España.

Ballesteros, F. 2003. Liebre de piornal, Lepus castroviejoi (Palacios, 1976). Galemys 15:3-13.

Ballesteros, F. 2007. Lepus castroviejoi (Palacios 1977). En: Palomo, L.J., Gisbert, J., Blanco, J.C. (eds.). Atlas y Libro Rojo de los Mamíferos Terrestres de España. Ministerio de Medio Ambiente, Madrid, España.

Ballesteros, F., Benito, J.L., González-Quirós, P. 1996. Situación de las poblaciones de liebres en el norte de la Península Ibérica. Quercus 128 : 12-17.

Ballesteros, F., Palacios, P. 2009. Situación y conservación de la liebre de Piornal Lepus castroviejoi en la Cordillera Cantábrica. Serie técnica Naturaleza y Parques Nacionales. Ministerio de Medio Ambiente y Medio Rural y Marino, Madrid, España.

Barnes, R.F.W., Tapper, S.C. 1985. A method for counting hares by spotlight. Journal of Zoology 206:273-276.

Barrientos, L.M. 1989. Situación del lobo en la provincia de Valladolid. Quercus 45: 22-26.
Bartolomé, D.J., Alonso, M.E., Pérez, J.A., Díez, C., Olmedo, J.A. 2004. Habitat selection of the Iberian hare (Lepus granatensis) in a plain agroenvironment depending on the time of the year. II World Lagomorph Conference (Vairao, Portugal).

Bonhomme, F., Fernández, J. Palácios, F., Catalán, J., Machordon, A. 1986. Caractérisation biochimique du complexe d'espèces du genre Lepus en Espagne. Mammalia 50(4):495-506.

Bray, Y., Marboutin, E., Péroux, R., Ferron, J. 2003. Reliability of stained placental scar counts in European hares. Wildlife Society Bulletin 31:237-245.

Cabrera, A. 1914. Fauna ibérica: mamíferos. Museo Nacional de Ciencias Naturales, Madrid, España. 441 pp.

Calzada, E., Martínez, F.J. 1994. Requerimientos y selección de hábitat de la liebre mediterránea (Lepus granatensis, Rosenhauer, 1856) en un paisaje agrícola mesetario. Ecología 8:381-394.

Carro, F. 2005. Historia natural de la liebre ibérica (Lepus granatensis, Rosenhauer 1856) en el Parque Nacional de Doñana (España). Tesis doctoral. Estación Biológica de Doñana.

Carro, F., Soriguer, R.C. 2002. Lepus granatensis (Rosenhauer,1856). En: Atlas de los Mamiferos Terrestres de España (ed. Palomo LJ, Gisbert J), pp. 452-455. Dirección General de Conservación de la NaturalezaSECEM-SECEMU, Madrid.

Carro, F., Beltrán, J.F., Márquez, F.J., Pérez, J.M., Soriguer, R.C. 2002. Supervivencia de la liebre ibérica (Lepus granatensis, Rosenhaeur, 1856) en el Parque Nacional de Doñana durante una época de inundaciones. Galemys 14(1):31-38.

Carro, F., Soriguer R.C. 2010. La liebre ibérica. Serie técnica Naturaleza y Parques Nacionales. Ministerio de Medio Ambiente y Medio Rural y Marino, Madrid, España.

Carro, F., Fernández, A., Soriguer, R., Castién, E. 2004. Population trends of the Iberian hare (Lepus granatensis) in the north and south of the Iberian Peninsula. Abstract book of the $2^{\text {nd }}$ World Lagomorph Conference, 26-31 Julio 2004, Vairao, Portugal. pp.124.

Castroviejo, J. 1970. Premières donnés sur l'écologie hivernale des vertébrés de la Cordillère Cantabrique. Alauda 38(2):126-149.

Delibes, M., Rodríguez, A., Ferreras, P. 2000. Action plan for conservation of the Iberian lynx (Lynx pardinus) in Europe. Convention for the Conservation of European Wildlife and Natural Habitats. A Large Carnivore Initiative for Europe. Strasbourg, Francia.

Díaz-Ruiz, F., Ferreras, P. 2013. Conocimiento científico sobre la gestión de depredadores generalistas en España: el caso del zorro (Vulpes vulpes) y la urraca (Pica pica). Ecosistemas 22(2):40-47.

Duarte, J. 2000. Liebre ibérica Lepus granatensis (Rosenhauer 1856). Galemys 12:3-14.

Duarte, J., Vargas, J.M. 1998. La perdrix rouge et le lièvre ibérique dans les oliveraies du sud de l'Espagne. Perspectives de gestion de ce type d'habitat. Bulletin Mensuel de I'Office National de la Chasse 236: 14-23.

Eiros-Bouza, J.M., Rodríguez-Torres, A. 1998. Tularemia. Revista Clínica Española 198:785-788.

Estonba, A,, Solís, A., Iriondo, M., Sanz-Martín, M.J., Pérez-Suárez, G., Markov, G., Palacios, F. 2006. The genetic distinctiveness of the three lberian hare species: Lepus europaeus, L. granatensis and L. castroviejoi. Mammalian Biology 71:52-59.

Farfán, M.A., Vargas, J.M., Real, R., Palomo, L.J., Duarte, J. 2004. Population parameters and reproductive biology of the Iberian hare Lepus granatensis in southern Iberia. Acta Theriologica 49:319-335.

Farfán, M.A., Duarte, J., Vargas, J.M. 2012. Effects of human induced landuse changes on the distribution of the Iberian hare. Journal of Zoology 286(2012)258-265.

Fernández, C., Purroy, F.J. 1990. Tendencias geográficas en la alimentación del Águila Real (Aquila chrysaetos L.) en Navarra. Ardeola 37(2):197-206.

Fernández, A., Soriguer, R., Castién, E., Carro, F. 2008. Reproduction parameters of the Iberian hare Lepus granatensis at the edge of its range. Wildlife Biology 14(4):434-443.

Fernández, A., Soriguer, R., Carro, F., Castién, E. 2010. Relation between physical condition and reproductive activity in a population of Iberian hares, Lepus granatensis in northern Iberian Peninsula. Folia Zoologica 59(1):1-8.

Fernández-Aguilar, X., Alzaga, V., Villanúa, D., Cabezón, O., García-Bocanegra, I., Dubey, J.P. Almería, S. 2013. Epidemiology and prevalence of Toxoplasma gondii infection in the Iberian hare (Lepus granatensis). Veterinary Parasitology 196 (1-2):194-198. 
Flux, J.E.C., Angermann, R. 1990. The hares and jackrabbits. En: Flux CJ y Angermann R (Eds). Rabbits, Hares and Pikas. Status Survey and Conservation Action Plan. Pp 61-94. IUCN, Suiza.

Freitas, H. 2006. Natural hybridization between the Iberian hare (Lepus granatensis) and the brown hare (L. europaeus) in northern Iberian Peninsula. MSc Thesis, Faculdade de Ciências da Univesidade do Porto, Porto, Portugal.

Frylestam, B. 1979. Structure, size and dynamics of three European hare populations in southern Sweden. Acta Theriologica 24:449-464.

Garrido, J.L. 2011. Estimación de aprovechamientos cinegéticos en España por especies y comunidades autónomas. Caza menor y caza mayor. FEDENCA-Real Federación Española de Caza, Madrid, España.

Gortázar, C., Millán, J., Acevedo, P., Escudero, M.A., Marco, J., Fernándezde Luco, D. 2007. A large-scale survey of brown hare Lepus europaeus and Iberian hare $L$. granatensis populations at the limit of their ranges. Wildlife Biology 13: 244-250.

Gortázar, C., Fernández-de Luco, D. 1995. La enfermedad hemorragica de la liebre. Trofeo 295:30-34.

Hansen, K. 1992. Reproduction in European hare in a Danish farmland. Acta Theriologica 37(1-2):27-40.

Jezierski, W. 1965. Studies on the European hare. VII. Changes in some elements of the structure and size of population. Acta Theriologica 10:11- 25.

Kauhala, K., Pekka, H. Y Hiltunen, M. 2005. Population dynamics of mountain hare Lepus timidus populations in Finland. Wildlife Biology 11(4):299-307.

Lavín, S., Blasco, J.M., Velarde, R., Mentaberre, G., Casas, E., Marin, C.M., Marco, I. 2006. Descripción del primer caso de Brucelosis en la Liebre Europea (Lepus europaeus) en la Península Ibérica. Información Veterinaria: Revista de la Organización Colegial Veterinaria Española 10:18-21.

López, J.M., Hernández, A., Purroy, F.J., Robles, J.L. 1996. Datos sobre la biología de la reproducción de la liebre ibérica (Lepus granatensis) en agrosistemas cerealistas de la provincia de León (NW de España). Revista Forestal 9(1):49-60.

López-Martínez, N. 1980. Les lagomorphs (Mammalia) du Pléistocène supérior de Jaurens. Nouveau Archives Museum d'Histoire Naturelle Lyon 18:5-16.

Melo-Ferreira, J., Boursot, P., Suchentrunk, F., Ferrand, N., Alves, P.C. 2005 Invasion from the cold past: extensive introgression of mountain hare (Lepus timidus) mitochondrial DNA into three other hare spoecies in northern Iberia. Molecular Ecology 14(8):2459-2464.

Melo-Ferreira, J., Boursot, P., Randi, E., Suchentrunk, F., Ferrand, N., Alves, P.C. 2007. The rise and fall of the mountain hare (Lepus timidus) during Pleistocene glaciations: expansion and retreat with hybridization in the Iberian Peninsula. Molecular Ecology 16:605-618.

Melo-Ferreira, J., Alves, P.C., Freitas, H., Ferrand, N,, Boursot, P. 2009. The genomic legacy from the extinct Lepus timidus to the three hare species of Iberia: contrast between mtDNA, sex chromosomes and autosomes. Molecular Ecology 18(12):2643-2658.

Palacios, F. 1976. Descripción de una nueva especie de liebre (Lepus castroviejoi) endémica de la Cordillera Cantábrica. Doñana. Acta Vertebrata 3(2):223-295.
Palacios, F. 1983. On the taxonomic status of the genus Lepus in Spain. Acta Zoologica Fennica 174:27-30.

Palacios, F. 1989. Biometric and morphologic features of the genus Lepus in Spain. Mammalia 53(2):227-264.

Palacios, F., Meijide, M. 1979. Distribución geográfica y hábitat de las liebres en la península ibérica. Naturalia Hispanica 19:1-40.

Palomo, L.J., Gisbert, J., Blanco, J.C. 2007. Atlas y Libro Rojo de los Mamíferos Terrestres de España. Ministerio de Medio Ambiente, Madrid.

Paupério, J., Alves, P.C. 2008. Diet of the Iberian hare (Lepus granatensis) in a mountain ecosystem. European Journal of Wildlife Research 54(4):571-579.

Pépin, D. 1989. Variations in survival of brown hare leverets from different farmland areas in the Paris basin. Journal of Applied Ecology 26:13-23.

Pérez-Suárez, G., Palacios, F., Boursot, P. 1994. Speciation and Paraphyly in Western Mediterranean Hares (Lepus castroviejoi, L. europaeus, $L$. granatensis, and $L$. capensis) Revealed by Mitochondrial DNA Phylogeny. Biochemical Genetics 32(11/12):423-436.

Pierpaoli, M., Riga, F., Trocchi, V., Randi, E. 1999. Species distinction and evolutionary relationships of the Italian hare (Lepus corsicanus) as described by mitochondrial DNA sequencing. Molecular Ecology 8:18051817.

Pikula, J., Beklová, M., Holesovská, Z., Trmel, F. 2004. Ecology of European brown hare and distribution of natural foci of tularaemia in the Czech Republic. Acta Veterinaria Brno 73:267-273.

Reynolds, J.C., O'Mahony, D., Aebischer, N.J. 2006. Implications of 'cyclical' population dynamics for the conservation of Irish hares (Lepus timidus hibernicus). Journal of Zoology 270(3):408-413.

Rodríguez-Alonso, M., Palacios-Alberti, J., Martín-Fernández, J.A., MartínGarcía, P., Sánchez-Cabrero, C., Naveso-Yelmo, M.A., Muñoz-Pulido, R., Yanes-García, T. 1997. La liebre. Ediciones Mundi-Prensa, MadridBarcelona-México.

Sánchez-García, C., Alonso, M.A., Bartolomé, D.J., Pérez, J.A., Larsen R.T., Gaudioso, V.R. 2012. Survival, home range patterns, probable causes of mortality, and den-site selection of the Iberian hare (Lepus, Leporidae, Mammalia) on arable farmland in north-west Spain. Italian Journal of Zoology iFirst: 1-8

Schmidt, N.M., Asferg, T., Forchhammer, M. 2004. Long-term patterns in European brown hare population dynamics in Denmark: effects of agriculture, predation and climate. BMC Ecology 4:15

Schneider, E., Leipoldt, M. 1983. DNA relationship within the genus Lepus in SW Europe. Acta Zoologica Fennica 174:31-33.

Stroh, G. 1931. Zwei sichere Altersmerkmale beim Hasen. Berliner Tieräztl 47:180-181.

Villafuerte, R., Viñuela, J. y Blanco, J.C. 1998. Extensive predator persecution caused by population crash in a game species: the case of Red kites and Rabbits in Spain. Biological conservation 84:181-188.

Villanúa, D., Pérez-Rodríguez, L., Casas, F., Alzaga, V., Acevedo, P., Viñuela, J., Gortazar, C. 2008. Sanitary risks of red-legged partridge releases: introduction of parasites. European Journal of Wildlife Research 54:199-204. 